\title{
Generalised Modified Taylor Series Approach of Developing $k$-step Block Methods for Solving Second Order Ordinary Differential Equations
}

\author{
Oluwaseun Adeyeye*, Zurni Omar \\ Department of Mathematics, School of Quantitative Sciences, University Utara Malaysia, Sintok, Kedah, Malaysia
}

Received October 6, 2020; Revised November 20, 2020; Accepted December 28, 2020

Cite This Paper in the following Citation Styles

(a): [1] Oluwaseun Adeyeye, Zurni Omar, "Generalised Modified Taylor Series Approach of Developing k-step Block Methods for Solving Second Order Ordinary Differential Equations," Mathematics and Statistics, Vol. 8, No. 6, pp. 773-781, 2020. DOI: 10.13189/ms.2020.080618.

(b): Oluwaseun Adeyeye, Zurni Omar , (2020). Generalised Modified Taylor Series Approach of Developing k-step Block Methods for Solving Second Order Ordinary Differential Equations. Mathematics and Statistics, 8(6), 773-781. DOI: 10.13189/ms.2020.080618.

Copyright $\mathrm{C} 2020$ by authors, all rights reserved. Authors agree that this article remains permanently open access under the terms of the Creative Commons Attribution License 4.0 International License

\begin{abstract}
Various algorithms have been proposed for developing block methods where the most adopted approach is the numerical integration and collocation approaches. However, there is another conventional approach known as the Taylor series approach, although it was utilised at inception for the development of linear multistep methods for first order differential equations. Thus, this article explores the adoption of this approach through the modification of the a forementioned conventional Taylor series approach. A new methodology is then presented for developing block methods, which is a more accurate method for solving second order ordinary differential equations, coined as the Modified Taylor Series (MTS) Approach. A further step is taken by presenting a generalised form of the MTS Approach that produces any $k$-step block method for solving second order ordinary differential equations. The computational complexity of this approach after being generalised to develop k-step block method for second order ordinary differential equations is calculated and the result shows that the generalised algorithm involves less computational burden, and hence is suitable for adoption when developing block methods for solving second order ordinary differential equations. Specifically, an alternate and easy-to-adopt approach to developing $k$-step block methods for solving second order ODEs with fewer computations has been introduced in this article with the developed block methods being suitable for solving second order differential equations directly.
\end{abstract}

Keywords Generalised, Modified Taylor Series, Block Method, Second Order, Ordinary Differential Equations, $k-$ step

\section{Introduction}

Block methods for the numerical solution of second order ordinary differential equations came to light in a bid to bypass the disadvantages of wastage in computational time of previously existing conventional methods $[1,2]$. To develop block methods, there are various approaches, with the most adopted approaches being the numerical integration approach as seen in studies by [3-6] and the interpolation approach as adopted by authors [7-9], amongst many other research work.

In the work by [10], these two approaches were displayed and the advantages and shortcomings were discussed. It was stated that although the derivation using integration approach is more complicated in comparison to interpolation approach, this approach is able to generalize the formulation of the integration coefficients while the interpolation approach fails in this regard. [11] however mentioned another approach for developing linear multistep methods which is the derivation by Taylor series. This approach is less rigorous to be adopted as seen in studies by [12,13]. However the methods developed by [12, 13] were restricted to the discrete schemes subject to boundary conditions, these methods were not further converted to block form and they were not adopted for initial value problems. The advantage of block methods is the ability of the method to simultaneously evaluate the method at different grid points for the equation under consideration.

Hence, this study will first give the general overview of how to adopt the Modified Taylor Series (MTS) Approach in obtaining the discrete schemes and the corresponding block methods. Then, the generalised form to develop any $k$-step block method is presented. The organization of this paper is in the format where Section 2 discusses the general approach for using the MTS approach to derive the two-step and three-step block methods, Section 3 gives the generalised form for developing any $k$-step 
block method and the computational complexity of this generalised form is also investigated. This generalised form is further verified by developing a four-step block method for solving second order ODEs and this paper is concluded in Section 4.

\section{Development of the Two-Step and Three-Step Block Methods Using MTS Ap- proach}

The development of block methods to solve second order ordinary differential equations of the form (1) below is considered

$$
y^{\prime \prime}=f\left(x, y, y^{\prime}\right)
$$

The main scheme for the block method is constructed from the general form of linear multistep methods

$$
y_{n+k}=\sum_{j=0}^{k-1} \alpha_{j} y_{n+j}+\sum_{j=0}^{k} \beta_{j} f_{n+j}
$$

Adopting Taylor series approximation to expand each of the terms in Equation (2) and then substituting the resulting expressions back in (2) gives:

$$
\begin{aligned}
& y\left(x_{n}\right)+(k h) y^{\prime}\left(x_{n}\right)+\frac{(k h)^{2}}{2 !} y^{\prime \prime}\left(x_{n}\right)+\cdots=\alpha_{0}\left[y\left(x_{n}\right)\right]+\alpha_{1}\left[y\left(x_{n}\right)+(h) y^{\prime}\left(x_{n}\right)+\frac{(h)^{2}}{2 !} y^{\prime \prime}\left(x_{n}\right)+\ldots\right] \\
& +\cdots+\alpha_{(k-1)}\left[y\left(x_{n}\right)+((k-1) h) y^{\prime}\left(x_{n}\right)+\frac{((k-1) h)^{2}}{2 !} y^{\prime \prime}\left(x_{n}\right)+\ldots\right]+\left[\beta_{0}\left[y^{(m)}\left(x_{n}\right)\right]+\beta_{1}\left[y^{(m)}\left(x_{n}\right)\right.\right. \\
& \left.+(h) y^{(m+1)}\left(x_{n}\right)+\frac{(h)^{2}}{2 !} y^{(m+2)}\left(x_{n}\right)+\ldots\right]+\cdots+\beta_{(k-1)}\left[y^{(m)}\left(x_{n}\right)+((k-1) h) y^{(m+1)}\left(x_{n}\right)\right. \\
& \left.\left.\quad+\frac{((k-1) h)^{2}}{2 !} y^{(m+2)}\left(x_{n}\right)+\ldots\right]+\beta_{k}\left[y^{(m)}\left(x_{n}\right)+(k h) y^{(m+1)}\left(x_{n}\right)+\frac{(k h)^{2}}{2 !} y^{(m+2)}\left(x_{n}\right)+\ldots\right]\right]
\end{aligned}
$$

Rewriting (3) in matrix form $A x=B$ while equating coefficients of $y^{(m)}\left(x_{n}\right)$ gives

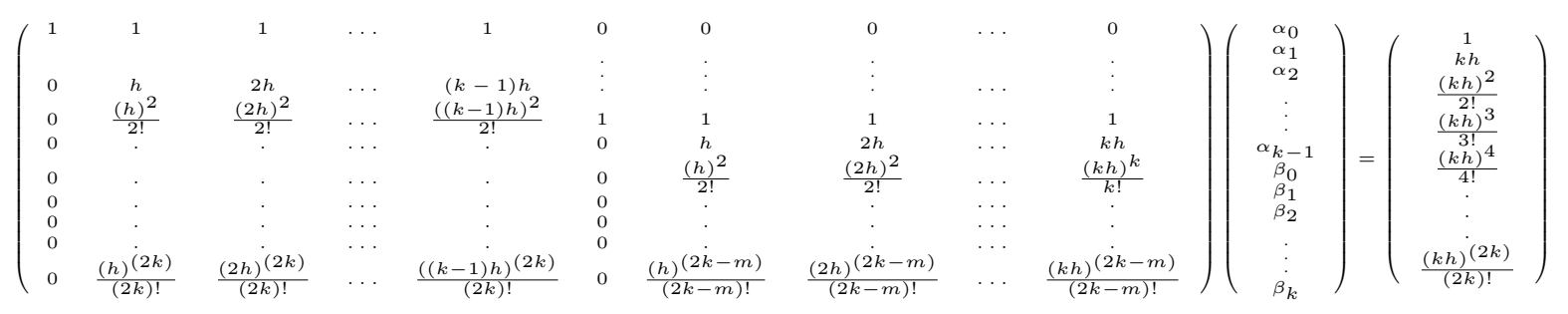

Using matrix inverse method to solve the system of equations yields the values of $\alpha_{0}, \alpha_{1}, \alpha_{2}, \ldots, \alpha_{k-1}$ and $\beta_{0}, \beta_{1}, \beta_{2}, \ldots, \beta_{k-1}, \beta_{k}$ and upon substitution back into (2) gives the desired linear multistep method.

The derivatives $y^{\prime}, y^{\prime \prime}, \ldots, y^{m-1}$ is taken at all grid points and the methods gotten together with the earlier multistep method are solved simultaneously to get the expected block method.

For further clarity, this approach is shown for the development of the two-step block method. The main scheme for the two-step block method is constructed from the general form of the linear multistep method of the form

$$
y_{n+k}=\sum_{j=0}^{k-1} \alpha_{j} y_{n+j}+\sum_{j=0}^{k} \beta_{j} f_{n+j}, \quad \text { where } k=2
$$

Adopting Taylor series approximation to expand each of the terms in Equation (4) and then substituting the resulting expressions back in (4) gives:

$$
\begin{aligned}
y\left(x_{n}\right)+(2 h) y^{\prime}\left(x_{n}\right)+\frac{(2 h)^{2}}{2 !} y^{\prime \prime}\left(x_{n}\right)+\frac{(2 h)^{3}}{3 !} y^{\prime \prime \prime}\left(x_{n}\right) & +\frac{(2 h)^{4}}{4 !} y^{i v}\left(x_{n}\right)=\alpha_{0}\left[y\left(x_{n}\right)\right]+\alpha_{1}\left[y\left(x_{n}\right)\right. \\
+(h) y^{\prime}\left(x_{n}\right)+\frac{(h)^{2}}{2 !} y^{\prime \prime}\left(x_{n}\right)+\frac{(h)^{3}}{3 !} y^{\prime \prime \prime}\left(x_{n}\right)+ & \left.\frac{(h)^{4}}{4 !} y^{i v}\left(x_{n}\right)\right]+\left[\beta_{0}\left[y^{\prime \prime}\left(x_{n}\right)\right]+\beta_{1}\left[y^{\prime \prime}\left(x_{n}\right)+(h) y^{\prime \prime \prime}\left(x_{n}\right)\right.\right. \\
& \left.+\frac{(h)^{2}}{2 !} y^{i v}\left(x_{n}\right)\right]+\beta_{2}\left[y^{\prime \prime}\left(x_{n}\right)+(2 h) y^{\prime \prime \prime}\left(x_{n}\right)+\frac{(2 h)^{2}}{2 !} y^{i v}\left(x_{n}\right)\right]
\end{aligned}
$$


Rewriting (5) in matrix form and equating coefficients of $y^{(m)}\left(x_{n}\right)$ yields

$$
\left(\begin{array}{ccccc}
1 & 1 & 0 & 0 & 0 \\
0 & h & 0 & 0 & 0 \\
0 & \frac{(h)^{2}}{2 !} & 1 & 1 & 1 \\
0 & \frac{(h)^{3}}{3 !} & 0 & h & 2 h \\
0 & \frac{(h)^{4}}{4 !} & 0 & \frac{(h)^{2}}{2 !} & \frac{(2 h)^{2}}{2 !}
\end{array}\right)\left(\begin{array}{c}
\alpha_{0} \\
\alpha_{1} \\
\beta_{0} \\
\beta_{1} \\
\beta_{2}
\end{array}\right)=\left(\begin{array}{c}
1 \\
2 h \\
\frac{(2 h)^{2}}{2 !} \\
\frac{(2 h)^{3}}{3 !} \\
\frac{(2 h)^{4}}{4 !}
\end{array}\right)
$$

Adopting matrix inverse method, the values of $\alpha_{0}, \alpha_{1}, \beta_{0}, \beta_{1}$ and $\beta_{2}$ are obtained as below

$$
\left(\begin{array}{c}
\alpha_{0} \\
\alpha_{1} \\
\beta_{0} \\
\beta_{1} \\
\beta_{2}
\end{array}\right)=\left(\begin{array}{c}
-1 \\
2 \\
\frac{h^{2}}{12} \\
\frac{5 h^{2}}{6} \\
\frac{h^{2}}{12}
\end{array}\right)
$$

Substituting (6) in (4) gives the discrete scheme

$$
y_{n+2}-2 y_{n+1}+y_{n}=\frac{h^{2}}{12}\left(f_{n+2}+10 f_{n+1}+f_{n}\right)
$$

Taking the first derivative of (4) at $x_{n}$, we expect the method

$$
y_{n}^{\prime}=\sum_{j=0}^{k-1} \alpha_{j} y_{n+j}+\sum_{j=0}^{k} \beta_{j} f_{n+j}, \quad \text { where } \quad k=2
$$

Adopting the same approach of the discrete scheme by taking the Taylor series expansion of (8), substituting the expansions back in (8) and then solving the corresponding matrix expression.

The coefficients $\alpha_{0}, \alpha_{1}, \beta_{0}, \beta_{1}$ and $\beta_{2}$ are found to be

$$
\left(\begin{array}{l}
\alpha_{0} \\
\alpha_{1} \\
\beta_{0} \\
\beta_{1} \\
\beta_{2}
\end{array}\right)=\left(\begin{array}{c}
-\frac{1}{h} \\
\frac{1}{h} \\
-\frac{7 h}{24} \\
-\frac{h}{4} \\
\frac{h}{24}
\end{array}\right)
$$

Substituting (9) in (8) gives

$$
y_{n}^{\prime}=\frac{1}{h}\left(-y_{n}+y_{n+1}\right)+\frac{h}{24}\left(-7 f_{n}-6 f_{n+1}+f_{n+2}\right)
$$

In the same vein, the derivatives at $x_{n+1}$ and $x_{n+2}$ are gotten to be

$$
\begin{aligned}
& y_{n+1}^{\prime}=\frac{1}{h}\left(-y_{n}+y_{n+1}\right)+\frac{h}{24}\left(3 f_{n}+10 f_{n+1}-f_{n+2}\right) \\
& y_{n+2}^{\prime}=\frac{1}{h}\left(-y_{n}+y_{n+1}\right)+\frac{h}{24}\left(f_{n}+26 f_{n+1}+9 f_{n+2}\right)
\end{aligned}
$$

Combining equations $(7),(10),(11)$ and (12) together gives the following equation which can be written in a matrix form

$$
\left(\begin{array}{cccc}
-2 & 1 & 0 & 0 \\
-\frac{1}{h} & 0 & 0 & 0 \\
-\frac{1}{h} & 0 & 1 & 0 \\
-\frac{1}{h} & 0 & 0 & 1
\end{array}\right)\left(\begin{array}{l}
y_{n+1} \\
y_{n+2} \\
y_{n+1}^{\prime} \\
y_{n+2}^{\prime}
\end{array}\right)=\left(\begin{array}{c}
-y_{n}+\frac{h^{2}}{12}\left(f_{n}+10 f_{n+1}+f_{n+2}\right) \\
-y_{n}^{\prime}-\frac{1}{h} y_{n}+\frac{h}{24}\left(-7 f_{n}-6 f_{n+1}+f_{n+2}\right) \\
-\frac{1}{h} y_{n}+\frac{h}{24}\left(3 f_{n}+10 f_{n+1}-f_{n+2}\right) \\
-\frac{1}{h} y_{n}+\frac{h}{24}\left(f_{n}+26 f_{n+1}+9 f_{n+2}\right)
\end{array}\right)
$$

Adopting matrix inverse method, $y_{n+1}, y_{n+2}, y_{n+1}^{\prime}$ and $y_{n+2}^{\prime}$ are determined as

$$
\left(\begin{array}{c}
y_{n+1} \\
y_{n+2} \\
y_{n+1}^{\prime} \\
y_{n+2}^{\prime}
\end{array}\right)=\left(\begin{array}{c}
y_{n}+h y_{n}^{\prime}+\frac{7}{24} h^{2} f_{n}+\frac{1}{4} h^{2} f_{n+1}-\frac{1}{24} h^{2} f_{n+2} \\
y_{n}+2 h y_{n}^{\prime}+\frac{2}{3} h^{2} f_{n}+\frac{4}{3} h^{2} f_{n+1} \\
y_{n}^{\prime}+\frac{5}{12} h f_{n}+\frac{2}{3} h f_{n+1}-\frac{1}{12} h f_{n+2} \\
y_{n}^{\prime}+\frac{1}{3} h f_{n}+\frac{4}{3} h f_{n+1}+\frac{1}{3} h f_{n+2}
\end{array}\right)
$$


which is equivalent to the following block,

$$
\begin{aligned}
& y_{n+1}=y_{n}+h y_{n}^{\prime}+\frac{h^{2}}{24}\left(7 f_{n}+6 f_{n+1}-f_{n+2}\right), \\
& y_{n+2}=y_{n}+2 h y_{n}^{\prime}+\frac{h^{2}}{3}\left(2 f_{n}+4 f_{n+1}\right) \\
& y_{n+1}^{\prime}=y_{n}^{\prime}+\frac{h}{12}\left(5 f_{n}+8 f_{n+1}-f_{n+2}\right) \\
& y_{n+2}^{\prime}=y_{n}^{\prime}+\frac{h}{3}\left(f_{n}+4 f_{n+1}+f_{n+2}\right) .
\end{aligned}
$$

The correctors of the block method (14) takes the form

$$
A^{0} Y_{n+k}=A^{1} Y_{n-k}+B^{1} Y_{n-k}^{\prime}+C^{0} Y_{n+k}^{\prime \prime}+C^{1} Y_{n-k}^{\prime \prime}
$$

where

$$
\begin{aligned}
& A^{0}=\left(\begin{array}{ll}
1 & 0 \\
0 & 1
\end{array}\right), \quad Y_{n+k}=\left(\begin{array}{c}
y_{n+1} \\
y_{n+2}
\end{array}\right), \quad A^{1}=\left(\begin{array}{cc}
0 & 1 \\
0 & 1
\end{array}\right), \quad Y_{n-k}=\left(\begin{array}{c}
y_{n-1} \\
y_{n}
\end{array}\right), \\
& B^{1}=\left(\begin{array}{cc}
0 & h \\
0 & 2 h
\end{array}\right), \quad Y_{n-k}^{\prime}=\left(\begin{array}{c}
y_{n-1}^{\prime} \\
y_{n}^{\prime}
\end{array}\right), \quad C^{0}=\left(\begin{array}{cc}
\frac{6 h^{2}}{24} & -\frac{h^{2}}{24} \\
\frac{4 h^{2}}{3} & 0
\end{array}\right), \\
& Y_{n+k}^{\prime \prime}=\left(\begin{array}{c}
f_{n+1} \\
f_{n+2}
\end{array}\right), \quad C^{1}=\left(\begin{array}{cc}
0 & \frac{7 h^{2}}{24} \\
0 & \frac{2 h^{2}}{3}
\end{array}\right) \quad \text { and } \quad Y_{n-k}^{\prime \prime}=\left(\begin{array}{c}
f_{n-1} \\
f_{n}
\end{array}\right)
\end{aligned}
$$

In addition, the MTS Approach is also adopted to develop the three-step block method. However, before following this procedure for the three-step method, the following conjecture is made.

Conjecture 2.1 The initial multistep scheme required to develop block method of the form (15) must have $\alpha_{j_{v}}-$ values equal to the order of the method to avoid an underdetermined system.

With reference to Conjecture 2.1, the scheme for the three-step block method can take the following forms: Form One:

$$
y_{n+3}=\alpha_{0} y_{n}+\alpha_{1} y_{n+1}+\sum_{j=0}^{3} \beta_{j} f_{n+j}
$$

Form Two:

$$
y_{n+3}=\alpha_{1} y_{n+1}+\alpha_{2} y_{n+2}+\sum_{j=0}^{3} \beta_{j} f_{n+j}
$$

Form Three:

$$
y_{n+3}=\alpha_{0} y_{n}+\alpha_{2} y_{n+2}+\sum_{j=0}^{3} \beta_{j} f_{n+j}
$$

Following the same process as with the two-step method, the discrete schemes, derivatives and the block form of each form is given as:

Method 1: Discrete Schemes and Derivatives

$$
\begin{aligned}
& y_{n+3}=-2 y_{n}+3 y_{n+1}+\frac{h^{2}}{12}\left(2 f_{n}+21 f_{n+1}+12 f_{n+2}+f_{n+3}\right), \\
& y_{n+2}=-y_{n}+2 y_{n+1}+\frac{h^{2}}{12}\left(f_{n}+10 f_{n+1}+f_{n+2}\right), \\
& y_{n}^{\prime}=\frac{1}{h}\left(-y_{n}+y_{n+1}\right)+\frac{h}{360}\left(-97 f_{n}-114 f_{n+1}+39 f_{n+2}-8 f_{n+3}\right), \\
& y_{n+1}^{\prime}=\frac{1}{h}\left(-y_{n}+y_{n+1}\right)+\frac{h}{360}\left(38 f_{n}+171 f_{n+1}-36 f_{n+2}+7 f_{n+3}\right), \\
& y_{n+2}^{\prime}=\frac{1}{h}\left(-y_{n}+y_{n+1}\right)+\frac{h}{360}\left(23 f_{n}+366 f_{n+1}+159 f_{n+2}-8 f_{n+3}\right), \\
& y_{n+3}^{\prime}=\frac{1}{h}\left(-y_{n}+y_{n+1}\right)+\frac{h}{360}\left(38 f_{n}+291 f_{n+1}+444 f_{n+2}+127 f_{n+3}\right) .
\end{aligned}
$$

Method 1: Block Form

$$
\begin{aligned}
& y_{n+1}=y_{n}+h y_{n}^{\prime}+\frac{h^{2}}{360}\left(97 f_{n}+114 f_{n+1}-39 f_{n+2}+8 f_{n+3}\right), \\
& y_{n+2}=y_{n}+2 h y_{n}^{\prime}+\frac{h^{2}}{45}\left(28 f_{n}+66 f_{n+1}-6 f_{n+2}+2 f_{n+3}\right), \\
& y_{n+3}=y_{n}+3 h y_{n}^{\prime}+\frac{h^{2}}{40}\left(39 f_{n}+108 f_{n+1}+27 f_{n+2}+6 f_{n+3}\right), \\
& y_{n+1}^{\prime}=y_{n}^{\prime}+\frac{h}{24}\left(9 f_{n}+19 f_{n+1}-5 f_{n+2}+f_{n+3}\right), \\
& y_{n+2}^{\prime}=y_{n}^{\prime}+\frac{h}{3}\left(f_{n}+4 f_{n+1}+4 f_{n+2}\right), \\
& y_{n+3}^{\prime}=y_{n}^{\prime}+\frac{h}{8}\left(3 f_{n}+9 f_{n+1}+9 f_{n+2}+3 f_{n+3}\right) .
\end{aligned}
$$

Method 2: Discrete Schemes and Derivatives 


$$
\begin{aligned}
& y_{n+3}=-y_{n+1}+2 y_{n+2}+\frac{h^{2}}{12}\left(f_{n+1}+10 f_{n+2}+f_{n+3}\right), \\
& y_{n}=2 y_{n+1}-y_{n+2}+\frac{h^{2}}{12}\left(f_{n}+10 f_{n+1}+f_{n+2}\right), \\
& y_{n}^{\prime}=\frac{1}{h}\left(-y_{n+1}+y_{n+2}\right)+\frac{h}{360}\left(-127 f_{n}-414 f_{n+1}+9 f_{n+2}-8 f_{n+3}\right), \\
& y_{n+1}^{\prime}=\frac{1}{h}\left(-y_{n+1}+y_{n+2}\right)+\frac{h}{360}\left(8 f_{n}-129 f_{n+1}-66 f_{n+2}+7 f_{n+3}\right), \\
& y_{n+2}^{\prime}=\frac{1}{h}\left(-y_{n+1}+y_{n+2}\right)+\frac{h}{360}\left(-7 f_{n}+66 f_{n+1}+129 f_{n+2}-8 f_{n+3}\right), \\
& y_{n+3}^{\prime}=\frac{1}{h}\left(-y_{n+1}+y_{n+2}\right)+\frac{h}{360}\left(8 f_{n}-9 f_{n+1}+414 f_{n+2}+127 f_{n+3}\right) .
\end{aligned}
$$

Method 2: Block Form

$$
\begin{aligned}
& y_{n+1}=y_{n}+h y_{n}^{\prime}+\frac{h^{2}}{360}\left(97 f_{n}+114 f_{n+1}-39 f_{n+2}+8 f_{n+3}\right), \\
& y_{n+2}=y_{n}+2 h y_{n}^{\prime}+\frac{h^{2}}{45}\left(28 f_{n}+66 f_{n+1}-6 f_{n+2}+2 f_{n+3}\right), \\
& y_{n+3}=y_{n}+3 h y_{n}^{\prime}+\frac{h^{2}}{40}\left(39 f_{n}+108 f_{n+1}+27 f_{n+2}+6 f_{n+3}\right), \\
& y_{n+1}^{\prime}=y_{n}^{\prime}+\frac{h}{24}\left(9 f_{n}+19 f_{n+1}-5 f_{n+2}+f_{n+3}\right), \\
& y_{n+2}^{\prime}=y_{n}^{\prime}+\frac{h}{3}\left(f_{n}+4 f_{n+1}+4 f_{n+2}\right), \\
& y_{n+3}^{\prime}=y_{n}^{\prime}+\frac{h}{8}\left(3 f_{n}+9 f_{n+1}+9 f_{n+2}+3 f_{n+3}\right) .
\end{aligned}
$$

Method 3: Discrete Schemes and Derivatives

$$
\begin{aligned}
& y_{n+3}=-\frac{1}{2} y_{n}+\frac{3}{2} y_{n+2}+\frac{h^{2}}{24}\left(f_{n}+12 f_{n+1}+21 f_{n+2}+2 f_{n+3}\right), \\
& y_{n+1}=\frac{1}{2} y_{n}+\frac{1}{2} y_{n+2}+\frac{h^{2}}{24}\left(-f_{n}-10 f_{n+1}-f_{n+2}\right), \\
& y_{n}^{\prime}=\frac{1}{2 h}\left(-y_{n}+y_{n+2}\right)+\frac{h}{45}\left(-14 f_{n}-33 f_{n+1}+3 f_{n+2}-f_{n+3}\right), \\
& y_{n+1}^{\prime}=\frac{1}{2 h}\left(-y_{n}+y_{n+2}\right)+\frac{h}{360}\left(23 f_{n}+21 f_{n+1}-51 f_{n+2}+7 f_{n+3}\right), \\
& y_{n+2}^{\prime}=\frac{1}{2 h}\left(-y_{n}+y_{n+2}\right)+\frac{h}{45}\left(f_{n}+27 f_{n+1}+18 f_{n+2}-f_{n+3}\right), \\
& y_{n+3}^{\prime}=\frac{1}{2 h}\left(-y_{n}+y_{n+2}\right)+\frac{h}{360}\left(23 f_{n}+141 f_{n+1}+429 f_{n+2}+127 f_{n+3}\right) .
\end{aligned}
$$

Method 3: Block Form

$$
\begin{aligned}
& y_{n+1}=y_{n}+h y_{n}^{\prime}+\frac{h^{2}}{360}\left(97 f_{n}+114 f_{n+1}-39 f_{n+2}+8 f_{n+3}\right), \\
& y_{n+2}=y_{n}+2 h y_{n}^{\prime}+\frac{h^{2}}{45}\left(28 f_{n}+66 f_{n+1}-6 f_{n+2}+2 f_{n+3}\right), \\
& y_{n+3}=y_{n}+3 h y_{n}^{\prime}+\frac{h^{2}}{40}\left(39 f_{n}+108 f_{n+1}+27 f_{n+2}+6 f_{n+3}\right), \\
& y_{n+1}^{\prime}=y_{n}^{\prime}+\frac{h}{24}\left(9 f_{n}+19 f_{n+1}-5 f_{n+2}+f_{n+3}\right), \\
& y_{n+2}^{\prime}=y_{n}^{\prime}+\frac{h}{3}\left(f_{n}+4 f_{n+1}+4 f_{n+2}\right), \\
& y_{n+3}^{\prime}=y_{n}^{\prime}+\frac{h}{8}\left(3 f_{n}+9 f_{n+1}+9 f_{n+2}+3 f_{n+3}\right) .
\end{aligned}
$$

It is observed from equations (20), (22) and (24) that all forms 1, 2 and 3 produced the same block form. The correctors of (20), (22) and (24) also takes the form (15) where

$$
\begin{aligned}
& A^{0}=\left(\begin{array}{ccc}
1 & 0 & 0 \\
0 & 1 & 0 \\
0 & 0 & 1
\end{array}\right), \quad Y_{n+k}=\left(\begin{array}{l}
y_{n+1} \\
y_{n+2} \\
y_{n+3}
\end{array}\right), \quad A^{1}=\left(\begin{array}{ccc}
0 & 0 & 1 \\
0 & 0 & 1 \\
0 & 0 & 1
\end{array}\right), \\
& Y_{n-k}=\left(\begin{array}{c}
y_{n-2} \\
y_{n-1} \\
y_{n}
\end{array}\right), \quad B^{1}=\left(\begin{array}{ccc}
0 & 0 & h \\
0 & 0 & 2 h \\
0 & 0 & 3 h
\end{array}\right), \quad Y_{n-k}^{\prime}=\left(\begin{array}{c}
y_{n-2}^{\prime} \\
y_{n-1}^{\prime} \\
y_{n}^{\prime}
\end{array}\right) \text {, } \\
& C^{0}=\left(\begin{array}{ccc}
\frac{19 h^{2}}{60} & -\frac{13 h^{2}}{120} & \frac{h^{2}}{45} \\
\frac{22 h^{2}}{15} & -\frac{2 h^{2}}{15} & \frac{2 h^{2}}{45} \\
\frac{27 h^{2}}{10} & \frac{27 h^{2}}{40} & \frac{3 h^{2}}{20}
\end{array}\right), \quad Y_{n+k}^{\prime \prime}=\left(\begin{array}{l}
f_{n+1} \\
f_{n+2} \\
f_{n+3}
\end{array}\right), \quad C^{1}=\left(\begin{array}{ccc}
0 & 0 & \frac{97 h^{2}}{360} \\
0 & 0 & \frac{28 h^{2}}{45} \\
0 & 0 & \frac{39 h^{2}}{40}
\end{array}\right) \\
& \text { and } Y_{n-k}^{\prime \prime}=\left(\begin{array}{c}
f_{n-2} \\
f_{n-1} \\
f_{n}
\end{array}\right)
\end{aligned}
$$

This leads to the following conjecture.

Conjecture 2.2 There is just one block method of the form $A^{0} Y_{n+k}=A^{1} Y_{n-k}+B^{1} Y_{n-k}^{\prime}+C^{0} Y_{n+k}^{\prime \prime}+C^{1} Y_{n-k}^{\prime \prime}$ associated with any $k$-step block method for solving a second order ordinary differential equation.

Therefore, since Conjecture 2.2 holds, a step can be taken further to obtain an algorithm for the MTS Approach for developing any $k$-step block method for second order ODEs. 


\section{Generalised MTS Approach for Developing $k$-step Block Method for Second Or- der ODEs}

Algorithm 3.1 shows the steps involved in adopting the MTS Approach to develop block methods of any step-length $k$ for solving second order ODEs.

\section{Algorithm 3.1}

START

Step 1: Compute the coefficient-values of the main scheme

$$
y_{n+k}=\sum_{j=0}^{k-1} \alpha_{j_{v}} y_{n+j}+\sum_{j=0}^{k} \beta_{j} f_{n+j}
$$

where $k$ is the step-length, $v=1,2$.

Step 2: Compute the coefficient-values of the schemes required in addition

$$
\begin{aligned}
y_{n+j_{3}} & =\sum_{j=0}^{k-1} \alpha_{j_{v}} y_{n+j}+\sum_{j=0}^{k} \beta_{j} f_{n+j} \\
y_{n+j_{4}} & =\sum_{j=0}^{k-1} \alpha_{j_{v}} y_{n+j}+\sum_{j=0}^{k} \beta_{j} f_{n+j} \\
\vdots & \\
y_{n+j_{k}}= & \sum_{j=0}^{k-1} \alpha_{j_{v}} y_{n+j}+\sum_{j=0}^{k} \beta_{j} f_{n+j}
\end{aligned}
$$

Step 3: Derive the coefficients of the first derivative schemes

$$
\begin{aligned}
y_{n}^{\prime} & =\sum_{j=0}^{k-1} \alpha_{j_{v}} y_{n+j}+\sum_{j=0}^{k} \beta_{j} f_{n+j} \\
y_{n+1}^{\prime} & =\sum_{j=0}^{k-1} \alpha_{j_{v}} y_{n+j}+\sum_{j=0}^{k} \beta_{j} f_{n+j} \\
y_{n+2}^{\prime} & =\sum_{j=0}^{k-1} \alpha_{j_{v}} y_{n+j}+\sum_{j=0}^{k} \beta_{j} f_{n+j} \\
\vdots & \sum_{j=0}^{k-1} \alpha_{j_{v}} y_{n+j}+\sum_{j=0}^{k} \beta_{j} f_{n+j}
\end{aligned}
$$

Step 4: Obtain a system of equation consisting of a combination of the resultant schemes from Steps 1-3 above, written in equivalent $A x=B$ matrix form, where

$x=\left(y_{n+1}, y_{n+2}, \ldots, y_{n+k}, y_{n+1}^{\prime}, y_{n+2}^{\prime}, \ldots, y_{n+k}^{\prime}\right)^{T}$

Step 5: Compute the coefficient-values of the required block method through matrix inverse solution of the system of equations in Step 4.

STOP

Specifically in Step 1 of Algorithm 3.1, the expected $\alpha_{j_{v}}$ are $\alpha_{j_{1}}$ and $\alpha_{j_{2}}$. Also, the major mathematical operations utilised in the steps of the algorithms are matrix inverse and matrix multiplication. Thus, to compute the computational complexity, the Big $(O)$ notation is adopted, which is useful to evaluate the complexity of an algorithm. The complexity involved for developing block method of any step-length $k$ for solving second order ODEs using the MTS Approach is presented in the table below.

Bearing in mind that taking the inverse of an $n \times n$ matrix involves a computational complexity of $O\left(n^{3}\right)$, while the matrix multiplication of an $n \times m$ matrix with an $m \times p$ matrix requires computational complexity of $O(n m p)$.

Table 1 details the step-by-step resultant computational complexity for the mathematical operations involved in Algorithm 3.1 . 
Table 1: $\operatorname{Big}(\mathrm{O})$ Notation Representation of the Computational Complexity for Algorithm 3.1

\begin{tabular}{|l|l|}
\hline Step & Computational Complexity $\mathbf{B i g}(\mathbf{O}))$ \\
\hline Step 1 & $O\left((k+3)^{2}\right)+O\left((k+3)^{3}\right)$ \\
\hline Step 2 & $(k-2)\left[O\left((k+3)^{2}\right)+O\left((k+3)^{3}\right)\right]$ \\
\hline Step 3 & $(k+1)\left[O\left((k+3)^{2}\right)+O\left((k+3)^{3}\right)\right]$ \\
\hline Step 4 & - \\
\hline Step 5 & $O\left((2 k)^{2}\right)+O\left((2 k)^{3}\right)$ \\
\hline$\Sigma=$ & $(2 k)\left[O\left((k+3)^{2}\right)+O\left((k+3)^{3}\right)\right]+\left[O\left((2 k)^{2}\right)+O\left((2 k)^{3}\right)\right]$ \\
\hline
\end{tabular}

A verification of this algorithm is conducted by developing the 4-step method using the MTS approach. Following Conjecture 2.1, the discrete schemes for the $k=4$ method can take different forms but just two will be considered to validate Conjecture 2.2 .

Form One for $k=4$

Following Algorithm 3.1, the resulting schemes for the steps are as follows

Step 1: Compute the coefficient-values of the main scheme

$$
y_{n+4}=-3 y_{n}+4 y_{n+1}+\frac{h^{2}}{120}\left(27 f_{n}+332 f_{n+1}+222 f_{n+2}+132 f_{n+3}+7 f_{n+4}\right)
$$

Step 2: Compute the coefficient-values of the schemes required in addition

$$
\begin{aligned}
& y_{n+2}=-y_{n}+2 y_{n+1}+\frac{h^{2}}{240}\left(19 f_{n}+204 f_{n+1}+14 f_{n+2}+4 f_{n+3}-f_{n+4}\right), \\
& y_{n+3}=-2 y_{n}+3 y_{n+1}+\frac{h^{2}}{240}\left(37 f_{n}+432 f_{n+1}+222 f_{n+2}+32 f_{n+3}-3 f_{n+4}\right),
\end{aligned}
$$

Step 3: Derive the coefficients of the first derivative schemes

$$
\begin{aligned}
& y_{n}^{\prime}=\frac{1}{h}\left(-y_{n}+y_{n+1}\right)+\frac{h}{360}\left(-367 f_{n}-540 f_{n+1}+282 f_{n+2}-116 f_{n+3}+21 f_{n+4}\right), \\
& y_{n+1}^{\prime}=\frac{1}{h}\left(-y_{n}+y_{n+1}\right)+\frac{h}{1440}\left(135 f_{n}+752 f_{n+1}-246 f_{n+2}+96 f_{n+3}-17 f_{n+4}\right), \\
& y_{n+2}^{\prime}=\frac{1}{h}\left(-y_{n}+y_{n+1}\right)+\frac{h}{1440}\left(97 f_{n}+1444 f_{n+1}+666 f_{n+2}-52 f_{n+3}+5 f_{n+4}\right), \\
& y_{n+3}^{\prime}=\frac{1}{h}\left(-y_{n}+y_{n+1}\right)+\frac{h}{1440}\left(119 f_{n}+1296 f_{n+1}+1578 f_{n+2}+640 f_{n+3}-33 f_{n+4}\right), \\
& y_{n+4}^{\prime}=\frac{1}{h}\left(-y_{n}+y_{n+1}\right)+\frac{h}{1440}\left(81 f_{n}+1508 f_{n+1}+1050 f_{n+2}+1932 f_{n+3}+469 f_{n+4}\right) .
\end{aligned}
$$

Step 4: Obtain a system of equation consisting of a combination of the resultant schemes from Steps 1-3 above, written in equivalent $A x=B$ matrix form, where $x=\left(y_{n+1}, y_{n+2}, \ldots, y_{n+k}, y_{n+1}^{\prime}, y_{n+2}^{\prime}, \ldots, y_{n+k}^{\prime}\right)^{T}$

Step 5: Compute the coefficient-values of the required block method through matrix inverse solution of the system of equations in Step 4 .

$$
\begin{aligned}
& y_{n+1}=y_{n}+h y_{n}^{\prime}+\frac{h^{2}}{1440}\left(367 f_{n}+540 f_{n+1}-282 f_{n+2}+116 f_{n+3}-21 f_{n+4}\right), \\
& y_{n+2}=y_{n}+2 h y_{n}^{\prime}+\frac{h^{2}}{90}\left(53 f_{n}+144 f_{n+1}-30 f_{n+2}+16 f_{n+3}-3 f_{n+4}\right), \\
& y_{n+3}=y_{n}+3 h y_{n}^{\prime}+\frac{h^{2}}{160}\left(147 f_{n}+468 f_{n+1}+54 f_{n+2}+60 f_{n+3}-9 f_{n+4}\right), \\
& y_{n+4}=y_{n}+4 h y_{n}^{\prime}+\frac{h^{2}}{45}\left(56 f_{n}+192 f_{n+1}+48 f_{n+2}+64 f_{n+3}\right), \\
& y_{n+1}^{\prime}=y_{n}^{\prime}+\frac{h}{720}\left(251 f_{n}+646 f_{n+1}-264 f_{n+2}+106 f_{n+3}-19 f_{n+4}\right), \\
& y_{n+2}^{\prime}=y_{n}^{\prime}+\frac{h}{90}\left(29 f_{n}+124 f_{n+1}+24 f_{n+2}+4 f_{n+3}-f_{n+4}\right), \\
& y_{n+3}^{\prime}=y_{n}^{\prime}+\frac{h}{80}\left(27 f_{n}+102 f_{n+1}+72 f_{n+2}+42 f_{n+3}-3 f_{n+4}\right), \\
& y_{n+4}^{\prime}=y_{n}^{\prime}+\frac{h}{45}\left(14 f_{n}+64 f_{n+1}+24 f_{n+2}+64 f_{n+3}+14 f_{n+4}\right) .
\end{aligned}
$$

\section{Form Two for $k=4$}

Following Algorithm 3.1, the resulting schemes for the steps are as follows

Step 1: Compute the coefficient-values of the main scheme

$$
y_{n+4}-2 y_{n+2}+y_{n}=\frac{h^{2}}{15}\left(f_{n+4}+16 f_{n+3}+26 f_{n+2}+16 f_{n+1}+f_{n}\right)
$$


Step 2: Compute the coefficient-values of the schemes required in addition

$$
\begin{aligned}
& y_{n+1}-\frac{1}{2} y_{n+2}-\frac{1}{2} y_{n}=\frac{h^{2}}{480}\left(f_{n+4}-4 f_{n+3}-14 f_{n+2}-204 f_{n+1}-19 f_{n}\right), \\
& y_{n+3}-\frac{3}{2} y_{n+2}+\frac{1}{2} y_{n}=\frac{h^{2}}{480}\left(-3 f_{n+4}+52 f_{n+3}+402 f_{n+2}+252 f_{n+1}+17 f_{n}\right),
\end{aligned}
$$

Step 3: Derive the coefficients of the first derivative schemes

$$
\begin{aligned}
& y_{n}^{\prime}=\frac{1}{h}\left(-\frac{1}{2} y_{n}+\frac{1}{2} y_{n+2}\right)+\frac{h}{180}\left(-53 f_{n}-144 f_{n+1}+30 f_{n+2}-16 f_{n+3}+3 f_{n+4}\right), \\
& y_{n+1}^{\prime}=\frac{1}{h}\left(-\frac{1}{2} y_{n}+\frac{1}{2} y_{n+2}\right)+\frac{h}{720}\left(39 f_{n}+70 f_{n+1}-144 f_{n+2}+42 f_{n+3}-7 f_{n+4}\right), \\
& y_{n+2}^{\prime}=\frac{1}{h}\left(-\frac{1}{2} y_{n}+\frac{1}{2} y_{n+2}\right)+\frac{h}{180}\left(5 f_{n}+104 f_{n+1}+78 f_{n+2}-8 f_{n+3}+f_{n+4}\right), \\
& y_{n+3}^{\prime}=\frac{1}{h}\left(-\frac{1}{2} y_{n}+\frac{1}{2} y_{n+2}\right)+\frac{h}{720}\left(31 f_{n}+342 f_{n+1}+768 f_{n+2}+314 f_{n+3}-15 f_{n+4}\right), \\
& y_{n+4}^{\prime}=\frac{1}{h}\left(-\frac{1}{2} y_{n}+\frac{1}{2} y_{n+2}\right)+\frac{h}{180}\left(3 f_{n}+112 f_{n+1}+126 f_{n+2}+240 f_{n+3}+59 f_{n+4}\right)
\end{aligned}
$$

Step 4: Obtain a system of equation consisting of a combination of the resultant schemes from Steps 1-3 above, written in equivalent $A x=B$ matrix form, where

$x=\left(y_{n+1}, y_{n+2}, \ldots, y_{n+k}, y_{n+1}^{\prime}, y_{n+2}^{\prime}, \ldots, y_{n+k}^{\prime}\right)^{T}$

Step 5: Compute the coefficient-values of the required block method through matrix inverse solution of the system of equations in Step 4 .

$$
\begin{aligned}
& y_{n+1}=y_{n}+h y_{n}^{\prime}+\frac{h^{2}}{1440}\left(367 f_{n}+540 f_{n+1}-282 f_{n+2}+116 f_{n+3}-21 f_{n+4}\right), \\
& y_{n+2}=y_{n}+2 h y_{n}^{\prime}+\frac{h^{2}}{90}\left(53 f_{n}+144 f_{n+1}-30 f_{n+2}+16 f_{n+3}-3 f_{n+4}\right), \\
& y_{n+3}=y_{n}+3 h y_{n}^{\prime}+\frac{h^{2}}{160}\left(147 f_{n}+468 f_{n+1}+54 f_{n+2}+60 f_{n+3}-9 f_{n+4}\right), \\
& y_{n+4}=y_{n}+4 h y_{n}^{\prime}+\frac{h^{2}}{45}\left(56 f_{n}+192 f_{n+1}+48 f_{n+2}+64 f_{n+3}\right), \\
& y_{n+1}^{\prime}=y_{n}^{\prime}+\frac{h}{720}\left(251 f_{n}+646 f_{n+1}-264 f_{n+2}+106 f_{n+3}-19 f_{n+4}\right), \\
& y_{n+2}^{\prime}=y_{n}^{\prime}+\frac{h}{90}\left(29 f_{n}+124 f_{n+1}+24 f_{n+2}+4 f_{n+3}-f_{n+4}\right), \\
& y_{n+3}^{\prime}=y_{n}^{\prime}+\frac{h}{80}\left(27 f_{n}+102 f_{n+1}+72 f_{n+2}+42 f_{n+3}-3 f_{n+4}\right), \\
& y_{n+4}^{\prime}=y_{n}^{\prime}+\frac{h}{45}\left(14 f_{n}+64 f_{n+1}+24 f_{n+2}+64 f_{n+3}+14 f_{n+4}\right) .
\end{aligned}
$$

Since equations (25) and (26) are same, Conjecture 2.2 is therefore validated.

\section{Conclusions}

This paper has presented a generalised form of the Modified Taylor Series Approach to develop $k$-step block methods for solving second order ODEs. The statements given in Conjecture 2.1 and 2.2 serve as a guide when developing the method and as seen in the development of the 4-step block method, the generalised form is valid and hence can be adopted for developing any $k$-step block method for solving second order ordinary differential equations. The calculation of the computational complexity is given to guide future work, in which a new methodology of developing block methods is presented, in making a comparison to this generalised form of the Modified Taylor Series Approach.

\section{REFERENCES}

[1] Awoyemi D. O., "A p-stable linear multistep method for solving general third order ordinary differential equations," International journal of computer mathematics, vol. 80, no. 8, pp. 985-991, 2003. DOI: 10.1080/0020716031000079572

[2] Butcher J. C., ’Numerical methods for ordinary differential equations,” New York: John Wiley \& Sons Inc, 2008.

[3] Omar Z., "Developing parallel 3-point implicit block method for solving second order ordinary differential equations directly," International journal of management studies, vol. 11, no. 1, pp. 91-103, 2004. URL: https://core.ac.uk/download/pdf/12115932.pdf

[4] Majid Z. A., Hasni M. M., Senu N., "Solving second order linear Dirichlet and Neumann boundary value problems by block method," IAENG International journal of applied mathematics, vol. 43, no. 2, pp. 71-76, 2013. URL: http://www.iaeng.org/IJAM/issues_ v43/issue_2/IJAM_ 43_2_ 04.pdf

[5] See P. P., Majid Z. A., Suleiman M., "Solving nonlinear two point boundary value problem using two step direct method," Journal of quality measurement and analysis, vol. 7, no. 1, pp. 127-138, 2011. URL: http://journalarticle.ukm.my/2897/1/jqma7-1-12-zanariah.pdf 
[6] Allogmany, R., Ismail, F., Ibrahim, Z. B., "Implicit two-point block method with third and fourth derivatives for solving general second order ODEs," Mathematics and Statistics, vol. 7, no. 4,pp. 116-123, 2019. DOI: 10.13189/ms.2019.070404

[7] Jator S. N., Li, J., "Solving two-point boundary value problems by a family of linear multistep methods," Neural, parallel and scientific computations, vol. 17, no. 2, pp. 135-146, 2009. URL: http://www.dynamicpublishers.com/Neural/NPSC2009/09NPSC.pdf

[8] Badmus A. M., Yahaya Y. A., "An accurate uniform order 6 block method for direct solution of general second order ordinary differential equations," Pacific journal of science and technology, vol. 10, no. 2, pp. 248-254, 2009. URL: http://www.akamaiuniversity.us/P 2_ 248.pdf

[9] Adesanya A. O., Odekunle M. R., Adeyeye A. O., "Continuous block hybrid predictor corrector method for the solution of $y^{\prime \prime}=f\left(x, y, y^{\prime}\right)$," International journal of mathematics and soft computing, vol. 2, no. 2, pp. 35-42, 2012. URL: https://www.intjmsc.com/articles/volume-2/issue-2

[10] Omar Z., Kuboye J. O., "Derivation of block methods for solving second order ordinary differential equations directly using direct integration and collocation approaches," Indian journal of science and technology, vol. 8, no. 12, pp. 1-4, 2015. DOI: $10.17485 / \mathrm{ijs} \mathrm{s} / 2015 / \mathrm{v} 8 \mathrm{i} 12 / 70646$

[11] Lambert J. D., ”Computational methods in ordinary differential equations,” London: Wiley, 1973.

[12] Li C. J., "A kind of multistep finite difference methods for arbitrary order linear boundary value problems," Applied mathematics and computation, vol. 196, no. 2, pp. 858-865, 2008. DOI: 10.1016/j.amc.2007.07.019

[13] Chen J., Li C., "High accuracy finite difference schemes for linear fourth order boundary value problem and derivatives," Journal of information and computational science, vol. 9, no. 10, pp. 2751-2759, 2012. URL: http://www.joics.com 